\title{
Relationships between attentional blink magnitude, RSVP target accuracy, and performance on other cognitive tasks
}

\author{
KAREN M. ARNELL and ASHLEY E. HOWE \\ Brock University, St. Catharines, Ontario, Canada \\ MARC F. JOANISSE \\ University of Western Ontario, London, Ontario, Canada \\ and \\ RAYMOND M. KLEIN \\ Dalhousie University, Halifax, Nova Scotia, Canada
}

\begin{abstract}
When two masked, to-be-attended targets are presented within approximately half a second of each other, performance on the second target (T2) suffers, relative to when the targets are presented further apart in time or when the first target (T1) can be ignored. This pattern of results is known as the attentional blink (AB). Typically, participants differ with respect to the magnitude of their $\mathrm{AB}$ and their overall target accuracy. Despite investigations as to what participant characteristics may influence $\mathrm{AB}$ performance (e.g., age, brain damage, or mood state), there has been no focused examination of whether individual differences in cognitive performance measures predict the magnitude of the $\mathrm{AB}$ or overall rapid serial visual presentation (RSVP) target accuracy. Our university student participants performed single-target and dual-target RSVP tasks, as well as a selection of cognitive tasks that did not use RSVP presentations, with color, letter, digit, and object stimuli. Overall performance on each of the RSVP targets (T1, T2, and single target) was predicted by speeded manual and vocal identification times to isolated stimuli and by performance with other RSVP targets. However, the magnitude of the $\mathrm{AB}$ was predicted only by $\mathrm{T} 1$ accuracy, not by any other performance measures. The results suggest that individual differences in AB magnitude do not result from differences in effective RSVP target encoding and are not well explained by varied information-processing abilities.
\end{abstract}

When two masked, to-be-attended targets are presented within approximately half a second of each other, performance on the second target (T2) is impaired, relative to when the targets are presented further apart in time or when the first target (T1) can be ignored (Broadbent \& Broadbent, 1987; Raymond, Shapiro, \& Arnell, 1992). This pattern of results is known as the attentional blink (AB; Raymond et al., 1992). The AB is most often investigated by embedding targets in rapid serial visual presentation (RSVP) streams in which stimuli are presented rapidly one at a time in the same spatial location. Dozens of studies have examined the presentation conditions that modulate the AB. For example, studies have examined the

The research was assisted by a grant from the Canadian Language and Literacy Research Network (CLLRNet) to K.M.A., M.F.J., and R.M.K. and by grants from NSERC, CFI, and OIT to K.M.A. Correspondence concerning this article should be addressed to K. M. Arnell, Department of Psychology, Brock University, St. Catharines, ON, L2S 3A1 Canada (e-mail: karnell@brocku.ca).

Note-This article was accepted by the previous editorial team, when Colin M. MacLeod was Editor. importance of masking T1 and T2 in order to produce an AB (e.g., Chun \& Potter, 1995; Giesbrecht \& Di Lollo, 1998; Grandison, Ghirardelli, \& Egeth, 1997; Raymond et al., 1992; Seiffert \& Di Lollo, 1997), the AB with targets from different modality combinations (e.g., Arnell \& Jolicour, 1999; Arnell \& Larson, 2002; Duncan, Martens, \& Ward, 1997; Hillstrom, Shapiro, \& Spence, 2002; Mondor, 1998; Potter, Chun, Banks, \& Muckenhoupt, 1998; Soto-Faraco \& Spence, 2002; Soto-Faraco et al., 2002), how the AB can be modulated by response selection demands (e.g., Arnell \& Duncan, 2002; Jolicœur, 1998, 1999; Jolicœur \& Dell'Acqua, 1999), and how the $\mathrm{AB}$ might vary when the difficulty of extracting the first target's identity is manipulated (McLaughlin, Shore, \& Klein, 2001; Shapiro, Raymond, \& Arnell, 1994; Shore, McLaughlin, \& Klein, 2001; Ward, Duncan, \& Shapiro, 1997). Other studies have examined the degree to which T2 was processed even when it could not be reported (e.g., Luck, Vogel, \& Shapiro, 1996; Shapiro, Driver, Ward, \& Sorensen, 1997; Vogel, Luck, \& Shapiro, 1998) or have investigated the nature of targets that can overcome the AB (e.g., Anderson, 2005; Keil \& Ihssen, 2004; Shapiro, Caldwell, \& Sorensen, 1997). 
In addition to explorations of procedural variables, some investigators have begun to look at group differences in $\mathrm{AB}$ magnitude when different participant populations are tested. For example, the $\mathrm{AB}$ has been shown to be larger for elderly participants (Lahar, Isaak, \& McArthur, 2001; Maciokas \& Crognale, 2003), schizophrenics (Cheung, Chen, Chen, Woo, \& Yee, 2002; Li et al., 2002), unilateral neglect patients (Husain, Shapiro, Martin, \& Kennard, 1997), ADHD patients (Li, Lin, Chang, \& Hung, 2004), Alzheimer's patients (Kavcic \& Duffy, 2003), and those reporting severe depression symptoms (Rokke, Arnell, Koch, \& Andrews, 2002), relative to matched controls. Green and Bavelier (2003) have also reported a reduced $\mathrm{AB}$ for action video game players. Typical university student participants also show variability in the magnitude of their $\mathrm{AB}$ and their overall target accuracy. It is somewhat surprising, then, that there has been no focused examination of whether individual differences in cognitive performance measures can predict individual differences in RSVP target accuracy or AB magnitude in this population. The question we will address here is the following: What cognitive performance measures might predict the magnitude of an individual's AB and his or her RSVP target accuracy?

Almost all theoretical models of the AB suggest that T2 performance suffers while limited capacity attentional resources are occupied with the processing of T1 (Shapiro, Arnell, \& Raymond, 1997). A popular class of these models postulates a bottleneck for conscious stimulus identification and/or consolidation in working memory where conscious identification and consolidation of T2's representation must wait until conscious identification and consolidation of T1 is complete (Arnell, Helion, Hurdelbrink, \& Pasieka, 2004; Chun \& Potter, 1995; Jolicœur, 1998, 1999; Jolicœur \& Dell'Acqua, 1998, 1999). If T2 is masked, its temporary representation will be overwritten by the mask while waiting for T1 to exit the bottleneck. If $\mathrm{T} 1$ processing in the bottlenecked stage(s) outlasts T2's representation, consolidation of T2 will fail, and subsequent $\mathrm{T} 2$ report accuracy will be reduced, resulting in an $\mathrm{AB}$. Therefore, bottleneck models predict that under dual-task conditions, more efficient consolidation of T1 should reduce the wait at the bottleneck, thereby reducing the AB. Assuming that an individual's T1 accuracy score reflects the effectiveness and efficiency of the individual's $\mathrm{T} 1$ processing, at least in part, one might expect a negative relationship between $\mathrm{T} 1$ accuracy and $\mathrm{AB}$ magnitude, where those with higher T1 accuracy would show smaller ABs. ${ }^{1}$ To the extent that those participants who efficiently and effectively encode $\mathrm{T} 1$ are the same participants who effectively and efficiently encode single RSVP targets, one might also predict that individuals who were better able to identify a single target in an RSVP stream of information would also show a smaller $\mathrm{AB}$ and have better performance on T1 and T2 on dual-task trials. Indeed, if RSVP target performance reflects the efficiency of identification and consolidation more generally, it is possible that faster and better identification of stimuli outside the
AB task (e.g., in speeded stimulus naming) would also predict improved RSVP target accuracy (T1, T2, or single target) and reduced $\mathrm{AB}$ magnitude.

Jolicœur's bottleneck model (e.g., Jolicœur, 1998, 1999; Jolicœur \& Dell'Acqua, 1998, 1999) also suggests that the processing resources needed for stimulus consolidation in working memory are the same as those required for response selection operations. Several studies have shown that when $\mathrm{T} 1$ requires a speeded response, the response selection requirements of the $\mathrm{T} 1$ task modulate the magnitude of the $\mathrm{AB}$, so that larger $\mathrm{ABs}$ are observed with greater T1 response selection requirements (Arnell \& Duncan, 2002; Jolicœur, 1998, 1999). Thus, to the extent that individuals differ in their response selection ability and the $A B$ is sensitive to such abilities, as postulated by Jolicœur (1998), it is reasonable to predict that individuals with longer response times (RTs) on a separate response selection task would produce larger $\mathrm{ABs}$ even when a nonspeeded $\mathrm{AB}$ paradigm is used. It is also reasonable to predict that individuals with longer RTs on a response selection task will have lower accuracy on T1, T2, or singletarget RSVP performance.

\section{The Present Study}

The goal of the present investigation was to examine individual differences in AB magnitude and RSVP target performance in relation to performance on several nonRSVP tasks that require identification of the same RSVP targets. Although previous research has provided a basis for several key predictions, studies to date have yet to directly examine these issues. Indeed, little direct evidence exists concerning the extent to which individual differences in AB magnitude or RSVP performance are associated with performance on other cognitive tasks.

In the present study, we measured the magnitude of the $\mathrm{AB}$ for each participant. We also measured each participant's overall accuracy for $\mathrm{T} 1$ and $\mathrm{T} 2$ in the $\mathrm{AB}$ task and his or her single-target RSVP accuracy across four stimulus types: letters, digits, objects, and colors. We predicted that individuals with higher single-target RSVP accuracy would also show higher T1 accuracy, higher T2 accuracy, and smaller AB magnitude, given that more efficient processing of single RSVP targets might predict more efficient processing of dual RSVP targets and that efficient processing of $\mathrm{T} 1$ and $\mathrm{T} 2$ in an $\mathrm{AB}$ task should reduce the likelihood that the $\mathrm{T} 2$ representation will be overwritten while waiting to be consolidated.

The participants in the present study also performed a variety of other non-RSVP tasks using the same stimulus items. These tasks required cognitive processes that have been implicated in RSVP performance and the AB. Vocal naming times (vocal RTs) to lone stimuli were measured, as were vocal naming times to $5 \times 10$ grids of stimuli (the rapid automatized naming, or RAN, task; Denckla \& Rudel, 1974). The RAN task provided a measure of naming times in the context of competing stimuli. The accuracy with which a participant could correctly identify which stimulus from an array had been briefly presented 
at a postcued location (the location probe task) was also measured. To the extent that rapid extraction of identity information from a stimulus is critical to RSVP target accuracy and/or AB magnitude, one might expect to see short naming times on vocal RT and RAN tasks, as well as high location probe accuracies associated with higher RSVP target accuracy and smaller ABs.

A manual RT task (pressing one of four keys matching the identity of the stimulus) was included to provide a measure of response selection speed. If response selection and stimulus consolidation do share a common bottlenecked processing stage (e.g., Jolicœur, 1998, 1999) and individuals differ in their response selection abilities, then to the degree that overall manual RTs reflected central processing demands, one would expect to see short response selection times associated with higher RSVP target accuracy and smaller ABs. A delayed RT task (in which the participants did not press the correct key until a tone was sounded after the stimulus) was also used to measure the speed of manual response execution without the identification and response selection components. This task was not expected to correlate with RSVP target accuracy or AB magnitude but was used simply as a motor execution control task. If response selection abilities, not response execution speed, underlie the expected relationships between manual RTs and $\mathrm{AB}$ magnitude and manual RTs and RSVP target accuracies, one would expect no relationship between delayed RTs and $\mathrm{AB}$ magnitude or RSVP target accuracy. However, if response execution speed underlies the expected relationships between manual RTs and AB magnitude and manual RTs and RSVP target accuracies, one would expect manual RTs and delayed RTs to be equally good predictors of AB magnitude and RSVP target accuracy.

\section{METHOD}

\section{Participants}

The present experiment draws on data from a previously conducted study (Arnell, Joanisse, Klein, Busseri, \& Tannock, 2006), conducted to better understand the relationship between reading performance and performance on the clinical RAN task. Sixty-four undergraduate students from Brock University $(n=34)$ and the University of Western Ontario $(n=30)$ received course credit or a small monetary payment for their participation. The participants ranged in age from 19 to 26 years, and all reported learning English before 8 years of age and having normal (or corrected-to-normal) visual acuity. Each participant performed the experiment individually in a single session lasting approximately $2 \mathrm{~h}$. All the participants performed the tasks in the following order: the RAN, manual RT, location probe, single-item vocal naming, delayed RT, single-target RSVP, and dual-target RSVP. The participants performed each task four times (once each with letters, digits, colors, and objects) in the same fixed order.

\section{Apparatus}

Stimulus presentation and response collection were controlled using E-Prime software (Schneider, Eschman, \& Zuccolotto, 2002) running on a Windows XP-based desktop PC with a 17-in. CRT color monitor. The participants made manual responses with the computer keyboard and vocal responses with a voice key integrated into a serial response box (Psychology Software Tools).

\section{Stimuli and Procedures}

RAN task. A 5-row $\times 10$-column grid of items was presented on the computer screen. Each grid contained colors (red, green, blue, and yellow), digits $(2,4,6$, and 9$)$, letters $(g, k, m$, and $r)$, or object pictures (dog, hand, book, and chair). Each grid measured approximately $25 \mathrm{~cm}$ wide $\times 18 \mathrm{~cm}$ high, subtending approximately $26.6^{\circ}$ of visual angle at an unfixed binocular viewing distance of approximately $50 \mathrm{~cm}$. Each individual element was approximately $1.5 \mathrm{~cm}$ high and wide, for a visual angle of approximately $1.7^{\circ}$. The participants pressed a key to display the grid and start the timer. The participants were instructed to accurately name each stimulus item as quickly as possible, beginning immediately after their keypress. The participants were told to name the grid items starting in the upper left element and ending at the lower right element, working their way across the rows from left to right. The participants pressed a key immediately after naming the last item to stop the computer timing. Items were named out loud, and errors were recorded by the experimenter. Each participant performed four trials, one with each stimulus type.

Manual RT task. A single stimulus from the RAN grid (e.g., a blue square) was presented in the center of the computer screen on each trial. The participants were instructed to press the key matching the identity of the stimulus as quickly and accurately as possible, using the index and middle fingers of both hands. Each trial began with a 500-msec presentation of a fixation cross and a 500-msec blank interval; then the stimulus was presented and remained on the screen until a response was made. A 500-msec intertrial interval followed the response. The "z," "x," "n," and "m" computer keyboard keys were labeled with the stimulus names to facilitate stimulus response mappings. Each participant performed one block for each of the four RAN stimulus categories (letters, digits, colors, and objects). Each block contained 48 trials, with each of the four stimulus exemplars presented 12 times each in random order (e.g., each of the four colors was presented 12 times in random order in the color block).

Delayed RT task. The delayed RT task was the same as the manual RT task, with the exception that the participants were told to delay their manual response until a tone sounded. The tone was randomly presented 1,500 or 2,000 msec after the onset of the stimulus element, thereby allowing the participant sufficient time to identify the stimulus and to select and prepare a response prior to the tone. The participants were instructed to prepare their response prior to the tone and then to make a speeded response as soon as the tone sounded. Each participant performed one block of 48 trials for each of the four stimulus categories.

Vocal naming task. The blocks, trials, and stimuli for the vocal naming task were identical to those for the manual RT task. However, in the vocal naming task, the participants reported the identity of the stimulus by vocally naming the stimulus into a microphone, instead of making a manual response. The stimulus remained on the screen until the vocal response had been detected. An experimenter recorded the accuracy and any spoiled trials (e.g., failure of the voice key) during the session. Each participant performed one block for each of the four stimulus categories.

Location probe task. On each trial, the participant viewed all four exemplars from a given stimulus category (e.g., all four colors), presented in random order in a row in the center of the computer screen. Each trial began with the presentation of a fixation cross for $500 \mathrm{msec}$, followed by a blank interval for $500 \mathrm{msec}$, and then the row of four stimuli for $125 \mathrm{msec}$. Immediately after the stimuli were removed from the screen a " $"$ " probe was presented randomly just below one of the four stimulus locations and remained on the screen until a response was made. The participant was asked to make an unspeeded response indicating which of the four exemplars had been presented in that location, guessing if unsure. Responses were made using the same keys and mappings as those used in the manual RT task. Each participant performed one block of 48 trials for each of the four stimulus categories. 
Single RSVP task. The participants were instructed to look for a specific target RAN element (e.g., the dog) in an RSVP stream of similar distractors and to report whether the target element was present or absent in the stream. Each trial began with a fixation cross that was presented for $500 \mathrm{msec}$ and a 500-msec blank screen; then an RSVP stream of 16 items was presented one at a time in the center of the computer screen. At the end of each stream, a sentence appeared that asked whether the target was present or absent in the stream. The participants made an unspeeded manual present/absent response. The target was present in the RSVP stream on two thirds of the trials and absent on one third of the trials. When present, the target was always the 6th or the 10th item in the stream. For object and color streams, each RSVP item was presented for $33 \mathrm{msec}$ and was followed by a $17-\mathrm{msec}$ blank interstimulus interval (ISI). For digit and letter streams, each item was presented for $50 \mathrm{msec}$ and was followed by a $17-\mathrm{msec}$ blank ISI. ${ }^{2}$ Six items were used as RSVP distractors for each stimulus category, but the same distractor was never presented in two successive positions within a stream. All the distractors had the same size, color, shading, and/or font as the targets from the same category. For color trials, distractor colors included the colors purple, orange, pink, brown, olive, and plum. For digit trials, distractors were the numbers $0,1,3,5,7$, and 8 . For letters, the distractors were $b, c, h, p, x$, and $y$; for objects, the distractor pictures were a teddy bear, a hat, a table, a wheelbarrow, a cup, and a fan. Each participant performed one block for each of the four stimulus categories. Each block contained 48 trials, which were divided into four sections of 12 trials each, so that every 12 trials, the target changed to the next stimulus in the set (e.g., for the first 12 trials in the color block, the participants searched for the red color, in the next 12 they searched for the blue color, etc.). The participants were informed when the target changed by a sentence, which appeared onscreen telling them the identity of the target for the next 12 trials. This sentence remained on the screen until a key was pressed.

Dual RSVP task. RSVP streams used for the dual-task trials were the same as those used for the single-task RSVP trials, with the following exceptions. One of the RSVP items was singled out from the others in the stream by virtue of a unique feature that was meant to attract attention. For the color RSVP trials, one of the colored squares contained an asterisk in the center, whereas all the others did not. The participants were instructed to identify the color of the square that contained the asterisk (blue, red, or yellow) for their first target task. For digit, letter, and object trials, this first target (T1) was colored red, whereas all the other RSVP items remained black. The participants were instructed to identify the red item for their first target task (the red item could be 2,4 , or 6 for digits; $g, k$, or $m$ for letters; and dog, hand, or chair for objects). After the RSVP stream, the participants were prompted to report the identity of T1 by presentation of a sentence onscreen. The participants identified T1 with an unspeeded buttonpress, using the labeled keys, and were told to guess if unsure. The second task on each trial was to report whether the fourth element on that block (i.e., the green color, the 9 , the $r$, or the book, which had not been used for T1) was present or absent in the RSVP stream anytime after the T1. After making their $\mathrm{T} 1$ response, the participants were prompted by a sentence on the computer screen to report whether this second target (T2) was present (press "1") or absent (press " 0 "), using an unspeeded response. Once the first and the second responses had both been entered, the next trial began after a 1 -sec blank intertrial interval. T1 was present on all trials as the sixth or eighth item in the RSVP stream. T2 was present on two thirds and absent on one third of all the trials. When present, T2 was presented equally often either two items after T1 or seven items after T1 in the RSVP stream. Each item in the color RSVP stream was presented for $66 \mathrm{msec}$, with a $17-\mathrm{msec}$ blank ISI. Each item in the digit, letter, and object streams was presented for $83 \mathrm{msec}$, with a 17-msec blank interval. Each participant performed one block of 48 trials for each of the four stimulus categories.

\section{RESULTS}

Mean performance measures for the single- and dual-task RSVP trials are presented in Tables 1 and 2 separately for each task and stimulus category. Single-task RSVP target performance is presented in terms of hit rate (the proportion of target-present trials on which the participant responded "present"), false alarm rate (the proportion of target-absent trials on which the participant responded "present"), and accuracy (the proportion of hits minus the proportion of false alarms). Similarly, dual-task RSVP T2 performance is presented in terms of hit rate, false alarm rate, and accuracy (hits - false alarms) separately for lag 2 and lag 7 and collapsed across lag. AB magnitude was calculated as T2 accuracy (hits - false alarms) at lag 2 subtracted from T2 accuracy (hits - false alarms) at lag 7. T1 accuracy was calculated as the proportion of trials on which the correct identity of T1 was reported. Proportion correct was used as the measure of $\mathrm{T} 1$ accuracy, given that a $\mathrm{T} 1$ was always present and the task was a three-alternative forced choice decision. Hits minus false alarms was used for $\mathrm{T} 2$ in the $\mathrm{AB}$ task and for single-target RSVP performance, given that the target task was a present/absent decision. Table 1 shows the accuracy scores used in the subsequent analyses, and Table 2 shows the hits and false alarms used to create the accuracy scores. All the trials were used for calculations of $\mathrm{T} 2$ accuracy and $\mathrm{AB}$ magnitude (not just $\mathrm{T} 1$ correct trials), so that the relationships between $\mathrm{T} 1$ and $\mathrm{T} 2$ accuracy and between $\mathrm{T} 1$ accuracy and the $\mathrm{AB}$ could also be examined. Although the following analyses are presented using singletarget and T2 accuracy defined as hits minus false alarms,

Table 1

Mean Accuracy for Dual- and Single-Target Rapid Serial Visual Presentation (RSVP) Tasks (With Standard Deviations) for Each Stimulus Type and Collapsed Across All Stimulus Types

\begin{tabular}{|c|c|c|c|c|c|c|c|c|c|c|c|c|}
\hline \multirow[b]{2}{*}{ Stimuli } & \multicolumn{2}{|c|}{$\begin{array}{c}\text { Single RSVP } \\
(\mathrm{H}-\mathrm{FA})\end{array}$} & \multicolumn{2}{|c|}{$\begin{array}{l}\text { Dual T1 } \\
\text { (Proportion } \\
\text { Correct) }\end{array}$} & \multicolumn{2}{|c|}{$\begin{array}{c}\text { Dual T2 } \\
\text { All Lags } \\
(\mathrm{H}-\mathrm{FA})\end{array}$} & \multicolumn{2}{|c|}{$\begin{array}{c}\text { Dual T2 } \\
\text { Lag } 2 \\
(\mathrm{H}-\mathrm{FA})\end{array}$} & \multicolumn{2}{|c|}{$\begin{array}{c}\text { Dual T2 } \\
\text { Lag } 7 \\
(\mathrm{H}-\mathrm{FA})\end{array}$} & \multicolumn{2}{|c|}{$\begin{array}{c}\text { Dual T2 } \\
\text { AB Magnitude } \\
\text { (Lag } 7-\text { Lag 2) } \\
\end{array}$} \\
\hline & $M$ & $S D$ & $M$ & $\overline{S D}$ & $M$ & $S D$ & $M$ & $S D$ & $M$ & $S D$ & $M$ & $S D$ \\
\hline Overall & .573 & .115 & .953 & .043 & .521 & .183 & .367 & .184 & .675 & .221 & .308 & .179 \\
\hline Colors & .661 & .158 & .929 & .105 & .694 & .272 & .619 & .306 & .771 & .293 & .151 & .251 \\
\hline Digits & .719 & .169 & .961 & .044 & .589 & .227 & .426 & .280 & .753 & .247 & .327 & .271 \\
\hline Letters & .590 & .165 & .958 & .046 & .426 & .243 & .252 & .267 & .600 & .291 & .348 & .273 \\
\hline Objects & .322 & .205 & .963 & .050 & .375 & .202 & .172 & .190 & .577 & .288 & .405 & .274 \\
\hline
\end{tabular}

Note-H - FA, hits minus false alarms; T1, first target; T2, second target; AB, attentional blink. 
Table 2

Mean Hits and False Alarms Used to Calculate Single-Target and Second Target (T2) Accuracies in Table 1

\begin{tabular}{|c|c|c|c|c|c|c|c|c|c|c|c|c|c|c|c|c|}
\hline \multirow[b]{2}{*}{ Stimuli } & \multicolumn{2}{|c|}{$\begin{array}{l}\text { Single } \\
\text { RSVP } \\
\text { Hits }\end{array}$} & \multicolumn{2}{|c|}{$\begin{array}{c}\text { Single } \\
\text { RSVP } \\
\text { False } \\
\text { Alarms }\end{array}$} & \multicolumn{2}{|c|}{$\begin{array}{l}\text { Dual T2 } \\
\text { Hits } \\
\text { All Lags }\end{array}$} & \multicolumn{2}{|c|}{$\begin{array}{l}\text { Dual T2 } \\
\text { False } \\
\text { Alarms } \\
\text { All Lags }\end{array}$} & \multicolumn{2}{|c|}{$\begin{array}{c}\text { Dual T2 } \\
\text { Hits } \\
\text { Lag } 2\end{array}$} & \multicolumn{2}{|c|}{$\begin{array}{l}\text { Dual T2 } \\
\text { False } \\
\text { Alarms } \\
\text { Lag } 2 \\
\end{array}$} & \multicolumn{2}{|c|}{$\begin{array}{c}\text { Dual T2 } \\
\text { Hits } \\
\text { Lag } 7\end{array}$} & \multicolumn{2}{|c|}{$\begin{array}{l}\text { Dual T2 } \\
\text { False } \\
\text { Alarms } \\
\text { Lag } 7\end{array}$} \\
\hline & $M$ & $S D$ & $M$ & $S D$ & $M$ & $S D$ & $M$ & $S D$ & $M$ & $S L$ & $M$ & $\overline{S D}$ & $M$ & $\overline{S D}$ & $M$ & $\overline{\mathrm{SD}}$ \\
\hline Overall & .727 & .109 & .154 & .094 & .651 & .146 & .130 & .100 & .498 & .176 & .130 & .099 & .805 & .167 & .130 & .099 \\
\hline olor & .849 & .120 & .188 & .123 & .772 & .200 & .077 & .111 & .696 & .250 & .07 & .1 & .848 & .222 & .077 & .111 \\
\hline Digits & .798 & .135 & .079 & .098 & .692 & .184 & .103 & .127 & .528 & .278 & .10 & .1 & .856 & .167 & .102 & .127 \\
\hline Letters & .758 & .131 & .168 & .155 & .586 & .188 & & .166 & .412 & .2 & & & .760 & .206 & .160 & .166 \\
\hline Objects & .503 & .209 & .181 & .174 & .556 & .195 & .181 & .163 & .354 & .233 & .181 & .163 & .759 & .244 & .181 & .163 \\
\hline
\end{tabular}

the same pattern of results was also observed when each participant's single-target and T2 RSVP performance was calculated in terms of $d^{\prime}$ or hit rate.

A significant $\mathrm{AB}$ was observed for each of the four stimulus types (all $p$ s $<.001$ for lag 2 vs. lag 7 T2 accuracy; see Table 1 for percentage of $A B$ magnitude for each stimulus type). For most of the subsequent analyses, scores on all the RSVP measures were averaged across the four stimulus categories (colors, digits, letters, and objects) to create a composite score for that measure (e.g., an average T2 accuracy score). As is evidenced by the results of principal components analyses, there were consistent individual differences in single-target RSVP accuracy, T1 accuracy, $\mathrm{T} 2$ accuracy, and $\mathrm{AB}$ magnitude across the stimulus types, with the exception of single-target color RSVP trials, which showed somewhat less variability. For example, with $\mathrm{AB}$ magnitude, a single component accounted for $45 \%$ of the variance among the four $\mathrm{AB}$ estimates (one for each stimulus type), and each $\mathrm{AB}$ measure loaded strongly on that component (loadings were .57-.76). Furthermore, the mean correlation across stimulus types (i.e., the average of the correlations for all stimulus type pairs) was .26 for $\mathrm{AB}$ magnitude. For $\mathrm{T} 2$ accuracy, the mean interstimulus correlation was .48, and a single component accounted for $61 \%$ of the variance in T2 accuracy, with strong loadings on that component for each stimulus type (.65-.86). For T1 accuracy, the mean interstimulus correlation was .34; a single component accounted for $53 \%$ of the variance in $\mathrm{T} 1$ accuracy, and loadings on that component ranged from .41 to .90 . For single-target RSVP accuracy, the mean interstimulus correlation was .24; a single component accounted for $45 \%$ of the variance in single-target accuracy, and loadings on that component varied from .37 to $.81 .^{3}$ Age and gender did not predict performance on any of the task measures (all $r \mathrm{~s}<.17$, all $p \mathrm{~s}>.18$ ).

\section{Associations Among RSVP Measures}

Table 3 shows the pattern of correlations between singletask RSVP accuracy, T1 accuracy, overall T2 accuracy, and AB magnitude for dual-task RSVP trials, averaged across the four stimulus types. As was predicted, the participants with higher accuracy in the single-target RSVP task also showed higher T2 accuracy in the dual task. However, in contrast to the predictions, neither singletarget accuracy nor $\mathrm{T} 2$ accuracy predicted $\mathrm{AB}$ magnitude significantly. AB magnitude was predicted by dual-task T1 accuracy, where the participants who had higher T1 accuracy showed a larger AB.

A simultaneous regression was performed in which single-target accuracy, $\mathrm{T} 1$ accuracy, and $\mathrm{AB}$ magnitude were entered as predictors of T2 accuracy across lags. Combined, the three predictors explained $50 \%$ of the variability in overall T2 accuracy $(R=.71, p<.001)$. Both single-target accuracy and T1 accuracy emerged as significant unique predictors of T2 accuracy (standardized regression coefficient $\beta=.48$, semipartial $r=.41, p<$ .001 , and $\beta=.31$, semipartial $r=.25, p<.01$, respectively). However, AB magnitude did not explain a significant amount of unique variability in overall T2 accuracy ( $\beta=.03$, semipartial $r=.02, p>.79$ ).

A simultaneous regression was then performed in which $\mathrm{AB}$ magnitude was the criterion and T1 accuracy, singletarget accuracy, and T2 accuracy were entered as predictors. The model explained $14 \%$ of the variability in $\mathrm{AB}$ magnitude $(R=.37, p<.05)$. Only $\mathrm{T} 1$ accuracy emerged as a significant unique predictor of $\mathrm{AB}$ magnitude $(\beta=$ .36 , semipartial $r=.28, p<.05)$. Neither single-target accuracy nor T2 accuracy was a significant unique predictor of AB magnitude $(\beta=-.03$, semipartial $r=-.02$, $p>.85$, and $\beta=.05$, semipartial $r=.03, p>.79$, respectively). Indeed, when T1 accuracy was removed as a

Table 3

Zero-Order Correlations Between Rapid Serial Visual Presentation (RSVP) Accuracy Scores

\begin{tabular}{|c|c|c|c|c|}
\hline & $\begin{array}{c}\text { T2 Accuracy } \\
(\operatorname{Lag} 2+\operatorname{Lag} 7)\end{array}$ & $\begin{array}{c}\text { T1 } \\
\text { Accuracy }\end{array}$ & $\begin{array}{c}\text { Single-Task } \\
\text { RSVP } \\
\text { Accuracy } \\
\end{array}$ & $\begin{array}{c}\text { AB } \\
\text { Magnitude } \\
(\operatorname{Lag} 7-\operatorname{Lag} 2) \\
\end{array}$ \\
\hline T1 accuracy & $.58^{* * *}$ & - & & \\
\hline Single-task RSVP accuracy & $.66^{* * *}$ & $.54^{* * *}$ & - & \\
\hline AB magnitude & .23 & $.37^{* *}$ & .19 & - \\
\hline
\end{tabular}


predictor, less than $5 \%$ of the variability in $\mathrm{AB}$ magnitude was explained by single-target and T2 accuracies. Overall, the results suggest that the magnitude of the $A B$ is unrelated to target detection ability (either when the target is presented in a single-task situation or when it is presented as T2 in a dual-task situation). In contrast, target accuracy in one situation (i.e., when the target is presented as a single target or as T1 or T2 on dual-target trials) predicts target accuracy in another situation.

To better understand the relationship between $\mathrm{T} 1 \mathrm{ac}-$ curacy and $\mathrm{AB}$ magnitude, $\mathrm{AB}$ magnitude was calculated using only trials on which T1 accuracy was correct (i.e., conditionalizing $\mathrm{T} 2$ accuracy on correct $\mathrm{T} 1$ accuracy, as is often done when $\mathrm{AB}$ data are analyzed). The $\mathrm{AB}$ magnitude on correct $\mathrm{T} 1$ trials was then correlated with $\mathrm{T} 1$ accuracy on all the trials. Using the conditionalized data, the correlation between $\mathrm{T} 1$ accuracy and $\mathrm{AB}$ magnitude was now eliminated $(r=.05, p>.65)$, suggesting that $\mathrm{T} 1$ accuracy predicts $\mathrm{AB}$ magnitude only due to $\mathrm{T} 1$ misses that reduce the potential for an $\mathrm{AB}$ (i.e., $\mathrm{T} 2$ should not be blinked if attention was never deployed to T1). ${ }^{4}$

\section{Associations Among RSVP Measures and Other Measures}

Mean performance measures for the non-RSVP tasks are presented in Table 4 separately for each task and stimulus category. For the manual RT, vocal RT, and delayed RT tasks, RTs are from correct trials only. Accuracy was at least $97 \%$ on these tasks. Fewer than $4 \%$ of the RTs were removed using the Van Selst and Jolicœur (1994) outlier elimination procedure.

Table 5 shows the pattern of correlations between singletask RSVP accuracy, T1 accuracy, T2 accuracy, AB magnitude, and measures from the non-RSVP tasks. T2, T1, and single-target accuracies were related significantly to manual RTs, vocal RTs, and RAN times. T1 accuracy was also related to probe location scores. In contrast, $\mathrm{AB}$ magnitude was not related to performance measures from any of the non-RSVP tasks.

A simultaneous regression was performed where all five non-RSVP measures (RAN times, manual, vocal and delayed RTs, and probe location scores) were entered as predictors of T2 accuracy. The combined predictors explained a significant $40 \%$ of the variability in T2 accuracy $(R=.64, p<.001)$. Manual RT was the only predictor to account for significant unique variability (semipartial $r=-.42, p<.001$; see Table 6 ), since much of the variability was shared by more than one predictor. When $\mathrm{T} 1$ accuracy, AB magnitude, and single-target accuracy were added in a second step, the variability accounted for increased significantly to $60 \%\left(R^{2}\right.$ change $=.20, p<$ $.001)$. Manual RT, single-target accuracy, and T1 accuracy emerged as significant unique predictors (semipartial $r=$ $-.28, p<.01$, semipartial $r=.28, p<.01$, and semipartial $r=.17, p<.05$, respectively; see Table 6). Note that when entered alone as predictors, the non-RSVP tasks accounted for $40 \%$ of the variability in T2 accuracy. This approximates the $50 \%$ accounted for by the other RSVP targets when they were entered alone.

When a simultaneous regression was performed in which all five non-RSVP measures were entered as predictors of $\mathrm{AB}$ magnitude, the combined predictors accounted for only $2 \%$ of the variability in AB magnitude $(R=.13, p>.96)$, with no predictor accounting for significant unique variability (all $p \mathrm{~s}>.37$; see Table 7). When T1 accuracy, T2 accuracy, and single-target accuracy were added in a second step, the variability increased significantly to $19 \%\left(R^{2}\right.$ change $\left.=.17, p<.05\right)$. T1 accuracy emerged as the only significant unique predictor (semipartial $r=.30, p<.05$; see Table 7).

\section{Correlations Between Measures for Individual Stimulus Types}

The analyses above were performed using data collapsed across the four stimulus types (colors, digits, letters, and objects). The use of the four stimulus types for each task also gives us an opportunity to examine the interrelationships among measures for each stimulus type individually. Despite the fact that there were fewer observations for each stimulus type than across stimulus types, the pattern of relationships was markedly similar for each stimulus type and overall across stimulus types. As was shown above, overall T2 accuracy (across stimulus types) was found to be related to T1 accuracy, singletarget RSVP accuracy, manual RTs, vocal RTs, and RAN times but was unrelated to delayed RTs, probe location accuracy, and AB magnitude. Replicating this pattern, T2 accuracy was also found to be significantly related to $\mathrm{T} 1 \mathrm{ac}-$ curacy $(r \mathrm{~s}=.23-.53)$, single-target RSVP accuracy $(r \mathrm{~s}=$ $.21-.59)$, manual RTs ( $r \mathrm{~s}=-.26$ to -.42$)$, and vocal RTs

Table 4

Mean Response Times (RTs, in Milliseconds) or Accuracy

(Proportion Correct) With Standard Deviations for Each Non-RSVP Task and Stimulus Combination

\begin{tabular}{|c|c|c|c|c|c|c|c|c|c|c|}
\hline \multirow[b]{2}{*}{ Stimuli } & \multicolumn{2}{|c|}{ RAN Times } & \multicolumn{2}{|c|}{$\begin{array}{c}\text { Manual } \\
\text { RTs }\end{array}$} & \multicolumn{2}{|c|}{$\begin{array}{l}\text { Vocal } \\
\text { RTs }\end{array}$} & \multicolumn{2}{|c|}{$\begin{array}{c}\text { Delayed } \\
\text { RTs }\end{array}$} & \multicolumn{2}{|c|}{$\begin{array}{c}\text { Probe } \\
\text { Localization }\end{array}$} \\
\hline & $M$ & $S D$ & $M$ & $S D$ & $M$ & $S D$ & $M$ & $S D$ & $M$ & $S D$ \\
\hline Overall & 25,251 & 3,717 & 642 & 113 & 454 & 56 & 305 & 76 & .939 & .034 \\
\hline Colors & 29,731 & 6,464 & 607 & 113 & 488 & 71 & 306 & 89 & .940 & .045 \\
\hline Digits & 19,223 & 3,099 & 544 & 80 & 414 & 60 & 304 & 82 & .938 & .058 \\
\hline Letters & 20,074 & 3,181 & 716 & 171 & 412 & 52 & 305 & 78 & .954 & .041 \\
\hline Objects & 31,974 & 4,806 & 700 & 159 & 504 & 66 & 304 & 84 & .923 & .061 \\
\hline
\end{tabular}

Note-RAN, rapid automatized naming. 
Table 5

Zero-Order Correlations Between Rapid Serial Visual Presentation Measures and Other Tasks

\begin{tabular}{lcccc}
\hline \multicolumn{5}{c}{ Measures and Other Tasks } \\
\multicolumn{1}{c}{$\begin{array}{c}\text { Single-Target } \\
\text { Accuracy }\end{array}$} & $\begin{array}{c}\text { T2 } \\
\text { Accuracy }\end{array}$ & $\begin{array}{c}\text { T1 } \\
\text { Accuracy }\end{array}$ & $\begin{array}{c}\text { AB } \\
\text { Magnitude }\end{array}$ \\
\hline RAN times & $-.40^{* * *}$ & $-.39^{* *}$ & $-.41^{* *}$ & .01 \\
Manual RT & $-.46^{* * *}$ & $-.56^{* * *}$ & $-.31^{*}$ & -.01 \\
Vocal RT & $-.49^{* * *}$ & $-.40^{* * *}$ & $-.43^{* *}$ & -.05 \\
Delayed RT & -.09 & -.18 & -.11 & -.03 \\
Location probe accuracy & .10 & -.23 & $.32^{*}$ & .12 \\
\hline
\end{tabular}

Note-T2, second target; T1, first target; AB, attentional blink; RAN, rapid automatized naming. ${ }^{*} p<.05 .{ }^{* *} p<.01 .{ }^{* * *} p<.001$.

$(r \mathrm{~s}=-.23$ to -.43$)$ for each of the four stimulus types individually. T2 accuracy was also significantly related to RAN times for two of the four stimuli (letter and object, $r \mathrm{~s}=-.30$ and -.51 , respectively) and was in the expected direction for digits and colors ( $r \mathrm{~s}=-.19$ and -.14 , respectively). Also, just as with the overall data, T2 accuracy was unrelated to delayed RT and probe location accuracy for any of the four stimulus types (all $p \mathrm{~s}>.05$ ). Thus, in the stimulus-specific analyses, 14 of the 16 possible correlations with T2 accuracy produced the significant effects that were also observed when the scores were collapsed across stimulus type, and 8 of 8 possible correlations with T2 accuracy produced the same null effects as those observed with the collapsed data.

Collapsed across stimulus type, $\mathrm{AB}$ magnitude was not related to any performance measures except $\mathrm{T} 1$ accuracy. This general pattern was also observed for each stimulus type. For each of the four stimulus types, AB magnitude was unrelated to manual RTs ( $r \mathrm{~s}=.04$ to .18$)$, delayed $\mathrm{RTs}(r \mathrm{~s}=-.17$ to .18$)$, vocal RTs $(r \mathrm{~s}=-.19$ to .13$)$, or probe location accuracy ( $r \mathrm{~s}=.02$ to .12 ). AB magnitude was also found to be unrelated to RAN performance except for color stimuli $(r=.27)$, unrelated to singletarget RSVP accuracy with the exception of object stimuli $(r=.36)$, and unrelated to T2 accuracy except for object stimuli $(r=.42)$. AB magnitude was significantly related to $\mathrm{T} 1$ accuracy for color and object stimuli $(r \mathrm{~s}=.35$ and .26 , respectively), but not for digits and letters $(r \mathrm{~s}=.08$ and -.01 , respectively). Thus, 25 of the 28 opportunities produced the same nonsignificant correlations with $\mathrm{AB}$ magnitude as those observed in the overall correlations collapsed across stimulus type, and two of the four stimulusspecific correlations produced the significant effects that were observed in the collapsed data. Overall, the highly similar results for each of the four stimulus types and the overall average suggests that the absence of significant relationships between $\mathrm{AB}$ magnitude and the other performance measures did not arise from the creation of a composite $\mathrm{AB}$ magnitude score averaged across stimulus types. Furthermore, the consistency in the pattern across stimulus types acts as a sort of within-experiment replication, which strengthens confidence in the overall results.

\section{DISCUSSION}

\section{Summary}

The predictors of $\mathrm{T} 2$ accuracy and $\mathrm{AB}$ magnitude in an $A B$ task were investigated. Correlations among RSVP target performance, $\mathrm{AB}$ magnitude, and performance on nonRSVP tasks were examined. Our results showed the following: (1) There was no significant relationship between

Table 6

Results of Simultaneous Regression Predicting Overall T2 Accuracy

\begin{tabular}{lccc}
\hline \multicolumn{1}{c}{ Measure } & $\begin{array}{c}\text { Standardized } \\
\text { Regression } \\
\text { Coefficients }(\beta)\end{array}$ & $\begin{array}{c}\text { Semipartial } \\
\text { Correlations }\end{array}$ & $p$ Values \\
\hline Step 1: Non-RSVP Predictors Only & & & \\
RAN times & -.20 & -.17 & .09 \\
Vocal RT & -.05 & -.04 & .71 \\
Manual RT & -.48 & -.42 & $<.001$ \\
Delayed RT & .02 & .02 & .86 \\
Location probe accuracy & .17 & .17 & .11 \\
Step 2: All Predictors & & & \\
RAN times & -.09 & -.07 & .41 \\
Vocal RT & .14 & .10 & .24 \\
Manual RT & -.34 & -.28 & .002 \\
Delayed RT & -.07 & -.06 & .46 \\
Location probe accuracy & .09 & .09 & .31 \\
AB magnitude & .06 & .06 & .52 \\
T1 accuracy & .24 & .17 & .05 \\
Single-target RSVP accuracy & .38 & .28 & .002 \\
\hline
\end{tabular}

Note-RSVP, rapid serial visual presentation; RAN, rapid automatized naming; $\mathrm{AB}$, attentional blink; $\mathrm{T} 1$, first target. 
Table 7

\begin{tabular}{|c|c|c|c|}
\hline Measure & $\begin{array}{c}\text { Standardized } \\
\text { Regression } \\
\text { Coefficients }(\beta)\end{array}$ & $\begin{array}{c}\text { Semipartial } \\
\text { Correlations }\end{array}$ & $p$ Values \\
\hline \multicolumn{4}{|l|}{ Step 1: Non-RSVP Predictors Only } \\
\hline RAN times & .05 & .04 & .76 \\
\hline Vocal RT & -.05 & -.04 & .77 \\
\hline Manual RT & .01 & .01 & .97 \\
\hline Delayed RT & .01 & .01 & .99 \\
\hline Location probe accuracy & .12 & .12 & .38 \\
\hline \multicolumn{4}{|l|}{ Step 2: All Predictors } \\
\hline RAN times & .17 & .14 & .26 \\
\hline Vocal RT & .09 & .06 & .63 \\
\hline Manual RT & .14 & .10 & .40 \\
\hline Delayed RT & -.06 & -.05 & .68 \\
\hline Location probe accuracy & -.01 & -.01 & .97 \\
\hline T2 accuracy & .13 & .08 & .52 \\
\hline T1 accuracy & .41 & .30 & .02 \\
\hline Single-target RSVP accuracy & .06 & .04 & .75 \\
\hline
\end{tabular}

Note- - RSVP, rapid serial visual presentation; RAN, rapid automatized naming; $\mathrm{T} 2$, second target; $\mathrm{T} 1$, first target.

AB magnitude and single-target or T2 accuracy, (2) high $\mathrm{T} 1$ accuracy was associated with a larger $\mathrm{AB}$ magnitude, (3) individual differences in RSVP target accuracy (T1, $\mathrm{T} 2$, or single target) were predicted by performance on manual RT, vocal RT, and RAN tasks, (4) non-RSVP tasks accounted for a total of $40 \%$ of the variability in T2 accuracy, and (5) individual differences in $\mathrm{AB}$ magnitude were not predicted by anything except $\mathrm{T} 1$ accuracy, and this relationship appeared to result simply from the fact that $\mathrm{T} 2$ was not blinked if $\mathrm{T} 1$ was unattended.

The results show that the magnitude of the $\mathrm{AB}$ is largely unrelated to individual differences in stimulus naming, response selection, and rapid stimulus identification and consolidation. In contrast, RSVP target performance (single or dual) is related to individual differences in tasks requiring rapid identification/consolidation and response selection. The participants with shorter manual RTs and shorter naming times showed greater RSVP target accuracy, but not larger $A B$ magnitudes. Thus, although one can predict individual RSVP target performance on the basis of these cognitive performance measures, individual $\mathrm{AB}$ magnitude cannot be predicted with these same measures, suggesting independence between single-target processing abilities and $\mathrm{AB}$ magnitude.

The finding that $\mathrm{AB}$ magnitude is not predicted by tasks requiring stimulus naming, response selection, and rapid stimulus identification and consolidation is, of course, a null effect, but it is not one that results from a lack of power. With the present sample size, relationships of about .25 or larger would be considered reliable, yet the correlations between $\mathrm{AB}$ magnitude and RAN times, vocal naming RTs, and manual RTs averaged .03, and the strongest of these relationships correlated at only .05. In contrast, the correlation between these three tasks and T2 accuracy averaged .45 . The difference in the ability of these tasks to predict $\mathrm{T} 2$ accuracy, but not $\mathrm{AB}$ magnitude, is especially provocative in light of the fact that $\mathrm{T} 2$ accuracy is the sum of $\mathrm{T} 2$ performance at lags 2 and 7, whereas $\mathrm{AB}$ magnitude is the difference between these same two performance measures. Thus, whereas the sum of the T2 accuracy measures across both lags is clearly related to the RAN, vocal RT, and manual RT tasks, the difference in these same measures is clearly unrelated. Furthermore, analyses indicated that the $\mathrm{AB}$ was a fairly stable individualdifference variable across the four stimulus types, with just under half of the variability in $\mathrm{AB}$ magnitudes shared by a single common factor (i.e., variability reflecting the $A B$ per se, separated from stimulus specific variability). This common variability was similar to the levels of common variability that were observed for T1 accuracy and singletarget RSVP accuracy. The latter two variables shared substantial correlations with many performance measures, whereas the $\mathrm{AB}$ did not. This suggests that differences in internal consistency of the RSVP variables do not underlie the pattern of relationships across tasks. Indeed, AB magnitude did correlate with a single variable (T1 accuracy), although this relationship simply suggested that no AB was observed when $\mathrm{T} 1$ was not attended.

\section{Predicting RSVP Target Accuracy}

Fairly strong positive relationships were observed between single-target accuracy, T1 accuracy, and T2 accuracy. These relationships were expected, given that all three tasks required the participants to extract and consolidate identity information from RSVP streams. However, the present results are the first that we know of to show positive associations and reliable individual differences in overall target performance across RSVP tasks.

Just as novel, but more surprising, was the finding that RSVP target performance (single target, T1, and T2) was significantly predicted by vocal naming times and manual RTs. The participants with higher RSVP target accuracy 
scores made faster manual responses to identify single stimuli and also named stimuli more rapidly. The manual RT and vocal naming tasks are very different from RSVP tasks, because speed (not accuracy) is the dependent variable and the stimuli are presented without distractors. Indeed, performance on these non-RSVP tasks predicted RSVP performance almost as well as did performance on other RSVP tasks.

The fact that vocal RTs to single stimuli predicted RSVP target accuracies as well as or better than the time to name an entire grid of stimuli suggests that the ability to select a target from a set of distractors prior to naming is not critical to the relationship between RAN times and RSVP target performance. The RAN task requires participants to track the stimuli spatiotemporally and to select the required stimulus from among 49 distractors that have recently been named or are soon to be named. This selection among distractors is not required in the vocal naming task, where a lone stimulus is presented for naming on each trial. Both the RAN task and the vocal RT task require participants to extract the identity of the to-benamed stimulus, consolidate it into working memory so that he or she is consciously aware of its identity, and then produce the vocal response. An online vocal response is not made in the RSVP tasks, so it is likely that performance on RSVP targets and naming times are related due to individual differences in the ability to rapidly identify and/or consolidate stimulus identities.

When T2 accuracy was predicted, regression results showed that manual RT was a unique predictor over and above vocal RT and RAN times, although all three were significant predictors when examined individually. Both vocal naming times and manual RT tasks require the participant to extract the identity of the stimulus and consolidate it into working memory. However, the manual RT task also requires participants to map the stimulus identity onto the appropriate key response (response selection) and then to execute the manual response. The unique explanatory power of the manual RT task suggests that response selection and/or execution abilities can explain additional variability in T2 accuracy scores.

Performance on the delayed RT task was unrelated to RSVP task performance (single target, T1, or T2). The delayed RT task has response execution requirements identical to those for the manual RT task. However, the long delay between the presentations of the stimulus and the tone removes individual differences in the speed of stimulus identification, consolidation, and response selection, since even the slowest participant will have completed these processes before the tone on the vast majority of trials. This makes the delayed RT task a purer measure of response execution. Therefore, the lack of a relationship between delayed RT and RSVP target accuracies suggests that it is not the response execution speed that accounts for the relationship between manual RT and RSVP target performance but, rather, the response selection speed.

Several studies have shown that when T1 requires a speeded response, increasing the response selection requirements of the T1 task increases the magnitude of the
AB (Arnell \& Duncan, 2002; Jolicœur \& Dell'Acqua, 1998, 1999). Jolicœur $(1998,1999)$ proposed a bottleneck model of the $\mathrm{AB}$ in which consolidation of $\mathrm{T} 2$ into working memory cannot proceed until consolidation of T1 has been completed. Jolicœur further suggested that the processing resources needed for stimulus consolidation in working memory are the same as those required for response selection operations. Thus, in Jolicœur's model, working memory consolidation and response selection efficiency modulate RSVP target accuracy and the magnitude of the AB. The present finding that individual differences in the ability to rapidly name items and select manual responses outside RSVP tasks predict target RSVP accuracy is consistent with Jolicœur's model. However, if one assumes that longer RTs on the manual RT task reflect longer consolidation and/or response selection times (at least in part), bottleneck models such as Jolicœur's would predict that short manual RTs would be associated with reduced $\mathrm{ABs}$, and this was not observed.

\section{Independence of AB Magnitude}

Intriguingly, there was no relationship between $\mathrm{AB}$ magnitude and overall performance on RSVP and non-RSVP tasks. If higher overall RSVP target accuracy suggests more efficient RSVP target processing and bottleneck models of the $\mathrm{AB}$ are correct that faster consolidation of T1 should reduce the wait at the bottleneck, one would expect a smaller $\mathrm{AB}$ for those with high single-target, $\mathrm{T} 1$, and $\mathrm{T} 2$ accuracy, as well as shorter naming and manual RTs. These relations were not observed.

The only variable that predicted $\mathrm{AB}$ magnitude was $\mathrm{T} 1$ accuracy, where the participants with higher $\mathrm{T} 1$ accuracy rates produced larger ABs. If high $\mathrm{T} 1$ accuracy suggests more efficient $\mathrm{T} 1$ processing, bottleneck models would suggest that individuals with higher T1 accuracy should show a smaller $\mathrm{AB}$, not a larger one. Indeed, manipulations of $\mathrm{T} 1$ difficulty have been shown to modulate the magnitude of the $\mathrm{AB}$ collapsed across participants, where more difficult T1 tasks produced larger ABs (e.g., Seiffert \& Di Lollo, 1997). However, McLaughlin et al. (2001) and Shore et al. (2001) suggested that individual differences in T1 performance are due primarily to the difference in resources allocated to $\mathrm{T} 1$ by different individuals, where more resources to $\mathrm{T} 1$ lead to better $\mathrm{T} 1$ performance and, thus, worse $\mathrm{T} 2$ accuracy in the $\mathrm{AB}$ window. Therefore, this model predicts that greater T1 accuracy will be associated with greater $\mathrm{AB}$ magnitude, as was observed here. However, in the present study, T1 accuracy and T2 accuracy were significantly and positively associated (see Table 3), suggesting that individual ability, not a T1/T2 resource trade-off, was responsible for the relationship between $\mathrm{T} 1$ accuracy and the $\mathrm{AB}$ magnitude. Also, if the positive association between $\mathrm{T} 1$ accuracy and $\mathrm{AB}$ magnitude results from some participants using more resources to process $\mathrm{T} 1$ at the expense of $\mathrm{T} 2$, as has been suggested by McLaughlin et al. (2001), the relationship between T1 accuracy and $\mathrm{AB}$ magnitude should become even stronger when the $\mathrm{AB}$ is calculated using only $\mathrm{T} 1$ correct trials, given that one can be sure that attentional resources were 
deployed to T1 on these trials. However, the relationship between $\mathrm{AB}$ magnitude and T1 accuracy was eliminated when $\mathrm{T} 2$ accuracy was conditionalized upon correct T1 report (i.e., there was no relationship between $\mathrm{T} 1 \mathrm{accu}-$ racy and $\mathrm{AB}$ magnitude when $\mathrm{T} 2$ accuracy was calculated using T1 correct trials only). Instead, the results suggest that $\mathrm{T} 1$ accuracy and $\mathrm{AB}$ magnitude were positively correlated simply because an $\mathrm{AB}$ could be observed only on trials in which $\mathrm{T} 1$ was properly attended (i.e., no AB should be observed when T1 was missed). Thus, participants who fail to attend to T1 more often will show lower T1 accuracy and a smaller $\mathrm{AB}$, unless all $\mathrm{T} 1$ misses are removed from the $\mathrm{AB}$ calculation.

\section{Conclusions}

This study is the first to look for relationships involving individual differences on tasks that are potential correlates of AB magnitude and RSVP target accuracy. Limitations in the processing efficiency of stimulus consolidation into working memory and/or response selection operations have been put forward as explanations of the $\mathrm{AB}$ (e.g., Chun \& Potter, 1995; Jolicœur, 1998, 1999; Jolicœur \& Dell'Acqua, 1998, 1999). Interestingly, individual differences on tasks requiring these mental processes were not found to predict individual differences in $\mathrm{AB}$ magnitude. However, individual differences in these tasks did predict single-target RSVP accuracy, T1 accuracy, and T2 accuracy averaged across lags. This leaves an intriguing situation in which it is not clear why some participants produce a large $\mathrm{AB}$ and others a small $\mathrm{AB}$ on the same task.

The tasks tested as correlates of the $\mathrm{AB}$ in the present study followed directly from $\mathrm{AB}$ models that stress the importance of stimulus identification, consolidation, and response selection limitations (e.g., Chun \& Potter, 1995; Jolicœur, 1999). It is, of course, possible, if not likely, that $\mathrm{AB}$ magnitude is related to other cognitive performance measures that were not tested here. For example, when fMRI scans were taken while participants performed an AB task, Marois, Chun, and Gore (2000) and Marcantoni, Lepage, Beaudoin, Bourgouin, and Richer (2003) observed involvement of the lateral frontal cortex (thought to be associated with working memory functioning; see, e.g., Owen, Evans, \& Petrides, 1996) and the anterior cingulate (linked to performance monitoring; see, e.g., Carter et al., 1998). Thus, individual differences in executive control and/or working memory measures may have some predictive utility and merit further investigation.

Interestingly, Olivers and Nieuwenhuis (2005) have shown recently that the magnitude of the $\mathrm{AB}$ was larger when participants were told to "try hard" during the AB task than when they were instructed to distract themselves with another mental activity. Although task instructions were manipulated between participants in Olivers and Nieuwenhuis's study, so that it is not possible to know how a given individual's $\mathrm{AB}$ magnitude changed across instruction conditions, the results raise the interesting possibility that the $\mathrm{AB}$ individuals produce on any given occasion have more to do with their temporary mental set and context (a state condition) than with their information- processing or attentional abilities (a trait condition). In the present study, we observed fairly substantial shared variance in $\mathrm{AB}$ magnitude across the four stimulus types, suggesting a relatively consistent $\mathrm{AB}$ magnitude. However, the four $\mathrm{AB}$ tasks were run back to back in a single session, so transient state factors (aside from stimulus type) are not likely to have varied greatly. Interestingly, however, McLaughlin et al. (2001) observed a correlation of +.66 when correlating $\mathrm{AB}$ magnitude found using the typical RSVP procedures with the same participants' AB magnitude found when removing all elements from the RSVP stream except the two targets and the items that immediately trailed them. The use of the different methodology and the fact that the two AB tasks were performed approximately 4 weeks apart suggest that the $\mathrm{AB}$ may be a stable within-subjects individual-differences characteristic when task instructions are kept constant. The present results suggest that $A B$ magnitude was roughly as stable across stimulus types as T1, T2, and single-target RSVP accuracy, which showed significant correlations with several of the cognitive performance measures used here. Therefore, we suggest that the stability of the AB observed here is sufficient to expect significant correlations with the other cognitive performance measures but that this expectation was not realized because $\mathrm{AB}$ magnitude is actually unrelated to these performance measures.

Existing models of the AB (e.g., Arnell et al., 2004; Chun \& Potter, 1995; Jolicœur, 1999) suggest that the AB reflects a fundamental information-processing bottleneck on conscious stimulus identification and consolidation. Such models then implicitly assume that the $\mathrm{AB}$ observed for a given individual depends on the information-processing abilities of the individual, which, aside from practice effects, should be more or less consistent for a given task and set of stimuli. However, the present results show that although these abilities are good predictors of an individual's RSVP target performance, they are not good predictors of an individual's AB magnitude - a pattern of results that is not consistent with an information-processing bottleneck. Bottleneck models appear to have much explanatory power when examined with the typical mean differences approach. Using a correlational, individualdifferences approach allows one to directly test models of the AB in a way that is different from the typical methodology of group means testing. This study is a first step in that direction. Its results suggest the need for future studies examining the stability of the $\mathrm{AB}$ within an individual and useful individual-difference predictors of $A B$ magnitude.

\section{REFERENCES}

Anderson, A. K. (2005). Affective influences on the attentional dynamics supporting awareness. Journal of Experimental Psychology: General, 134, 258-281.

ARNELL, K. M., \& DUNCAN, J. (2002). Shared and separate sources of dual-task cost in stimulus identification and response selection. Cognitive Psychology, 44, 105-147.

Arnell, K. M., Helion, A. M., Hurdelbrink, J. A., \& PasiekA, B. (2004). Dissociating sources of dual-task interference using human electrophysiology. Psychonomic Bulletin \& Review, 11, 77-83. 
Arnell, K. M., Joanisse, M. F., Klein, R. M., Busseri, M. A., \& TANNOCK, R. (2006). Decomposing RAN: The contribution of object recognition and attention to rapid naming and reading. Manuscript submitted for publication.

Arnell, K. M., \& Jolicœur, P. (1999). The attentional blink across stimulus modalities: Evidence for central processing limitations. Journal of Experimental Psychology: Human Perception \& Performance, 25, 630-648.

ARNELL, K. M., \& LARSON, J. M. (2002). Cross-modality attentional blinks without preparatory task-set switching. Psychonomic Bulletin \& Review, 9, 497-506.

Broadbent, D. E., \& Broadbent, M. H. P. (1987). From detection to identification: Response to multiple targets in rapid serial visual presentation. Perception \& Psychophysics, 42, 105-113.

Carter, C. S., BraVer, T. S., BarCh, D. M., BotVinick, M. M., Noll, D., \& Cohen, J. D. (1998). Anterior cingulate cortex, error detection, and the online monitoring of performance. Science, 280, 747-749.

Cheung, V., Chen, E. Y. H., Chen, R. Y. L., Woo, M. F., \& Yee, B. K. (2002). A comparison between schizophrenia patients and healthy controls on the expression of attentional blink in a rapid serial visual presentation (RSVP) paradigm. Schizophrenia Bulletin, 28, 443-458.

Chun, M. M., \& Potter, M. C. (1995). A two-stage model for multiple target detection in rapid serial visual presentation. Journal of Experimental Psychology: Human Perception \& Performance, 21, 109-127.

DENCKLA, M. B., \& RUDEL, R. [G.] (1974). Rapid "automatized" naming of pictured objects, colors, letters and numbers by normal children. Cortex, 10, 186-202.

DunCAN, J., MARTENS, S., \&WARD, R. (1997). Restricted attentional capacity within but not between sensory modalities. Nature, $\mathbf{3 8 7}, 808-810$.

GieSBRECHT, B. L., \& Di Lollo, V. (1998). Beyond the attentional blink: Visual masking by item substitution. Journal of Experimental Psychology: Human Perception \& Performance, 24, 1454-1466.

Grandison, T. D., Ghirardelli, T. G., \& EgETH, H. E. (1997). Beyond similarity: Masking of the target is sufficient to cause the attentional blink. Perception \& Psychophysics, 59, 266-274.

Green, C. S., \& BAVElier, D. (2003). Action video game modifies visual selective attention. Nature, 423, 534-537.

Hillstrom, A. P., Shapiro, K. L., \& Spence, C. (2002). Attentional limitations in processing sequentially presented vibrotactile targets. Perception \& Psychophysics, 64, 1068-1082.

Husain, M., Shapiro, K., Martin, J., \& KenNard, C. (1997). Abnormal temporal dynamics of visual attention in spatial neglect patients. Nature, 385, 154-156.

JoLICEUR, P. (1998). Modulation of the attentional blink by on-line response selection: Evidence from speeded and unspeeded Task deci- $^{-}$ sions. Memory \& Cognition, 26, 1014-1032.

JolicceuR, P. (1999). Concurrent response selection demands modulate the attentional blink. Journal of Experimental Psychology: Human Perception \& Performance, 25, 1097-1113.

JoLICEUR, P., \& DELL'ACQUA, R. (1998). The demonstration of shortterm consolidation. Cognitive Psychology, 36, 138-202.

Jolicceur, P., \& Dell'ACQUA, R. (1999). Attentional and structural constraints on memory encoding. Psychological Research, 62, 154-164.

KaVCic, V., \& Duffy, C. J. (2003). Attentional dynamics and visual perception: Mechanisms of spatial disorientation in Alzheimer's disease. Brain, 126, 1173-1181.

KeIL, A., \& IHSSEN, N. (2004). Identification facilitation for emotionally arousing verbs during the attentional blink. Emotion, 4, 23-35.

LAHAR, C. J., IsAAK, M. I., \& MCArthUR, A. D. (2001). Age differences in the magnitude of the attentional blink. Aging, Neuropsychology, \& Cognition, 8, 149-159.

LI, C. R., LIN, W., Chang, H., \& Hung, Y. (2004). A psychophysical measure of attention deficit in children with attention-deficit/ hyperactivity disorder. Journal of Abnormal Psychology, 113, 228-236.

Li, C. R., Lin, W., YAng, Y., HuAng, C., Chen, T., \& Chen, Y. (2002). Impairment of temporal attention in patients with schizophrenia. NeuroReport, 13, 1427-1430.

Luck, S. J., Vogel, E. K., \& Shapiro, K. L. (1996). Word meanings can be accessed but not reported during the attentional blink. Nature, 383, 616-618.
Maciokas, J. B., \& Crognale, M. A. (2003). Cognitive and attentional changes with age: Evidence from attentional blink deficits. Experimental Aging Research, 29, 137-153.

Marcantoni, W. S., LePage, M., Beaudoin, G., Bourgouin, P., \& RICHER, F. (2003). Neural correlates of dual task interference in rapid visual streams: An fMRI study. Brain \& Cognition, 53, 318-321.

Marois, R., Chun, M. M., \& Gore, J. (2000). Neural correlates of the attentional blink. Neuron, 28, 299-308.

Mclaughlin, E. N., Shore, D. I., \& Klein, R. M. (2001). The attentional blink is immune to masking induced data limits. Quarterly Journal of Experimental Psychology, 54A, 169-196.

Mondor, T. A. (1998). A transient processing deficit following selection of an auditory target. Psychonomic Bulletin \& Review, 5, 305-311.

Olivers, C. N. L., \& NiEUWENHUIS, S. (2005). The beneficial effect of concurrent task-irrelevant mental activity on temporal attention. Psychological Science, 16, 265-269.

Owen, A. M., Evans, A. C., \& PeTrides, M. (1996). Evidence for a two-stage model of spatial working memory processing within the lateral frontal cortex: A positron emission tomography study. Cerebral Cortex, 6, 31-38.

Potter, M. C., Chun, M. M., Banks, B. S., \& Muckenhoupt, M. (1998). Two attentional deficits in serial target search: The visual attentional blink and an amodal task-switch operation. Journal of Experimental Psychology: Learning, Memory, \& Cognition, 24, 979992.

RAYMOND, J. E., Shapiro, K. L., \& Arnell, K. M. (1992). Temporary suppression of visual processing in an RSVP task: An attentional blink? Journal of Experimental Psychology: Human Perception \& Performance, 18, 849-860.

RoKKe, P. D., ARNell, K. M., Koch, M. D., \& Andrews, J. T. (2002). Dual-task attention deficits in dysphoric mood. Journal of Abnormal Psychology, 111, 370-379.

Schneider, W., Eschman, A., \& Zuccolotto, A. (2002). E-Prime user's guide. Pittsburgh: Psychology Software Tools.

Seiffert, A. E., \& Di Lollo, V. (1997). Low-level masking and the attentional blink. Journal of Experimental Psychology: Human Perception \& Performance, 23, 1061-1073.

ShAPIRO, K. L., ARNELl, K. M., \& RAYMOnd, J. E. (1997). The attentional blink. Trends in Cognitive Sciences, 1, 291-296.

Shapiro, K. L., Caldwell, J., \& Sorensen, R. E. (1997). Personal names and the attentional blink: A visual "cocktail party" effect. Journal of Experimental Psychology: Human Perception \& Performance, 23, 504-514.

ShAPIRO, K. L., Driver, J., WARd, R., \& Sorensen, R. E. (1997). Priming from the attentional blink: A failure to extract visual tokens but not visual types. Psychological Science, 8, 95-100.

Shapiro, K. L., RAYMOND, J. E., \& ARNELL, K. M. (1994). Attention to visual pattern information produces the attentional blink in rapid serial visual presentation. Journal of Experimental Psychology: Human Perception \& Performance, 20,357-371.

ShORE, D. I., McLaughlin, E. N., \& KLEIN, R. M. (2001). Modulation of the attentional blink by differential resource allocation. Canadian Journal of Experimental Psychology, 55, 318-324.

SoTO-FARACO, S., \& SPENCE, C. (2002). Modality-specific auditory and visual temporal processing deficits. Quarterly Journal of Experimental Psychology, 55A, 23-40.

Soto-Faraco, S., Spence, C., Fairbank, K., Kingstone, A., HillStrom, A. P., \& Shapiro, K. (2002). A crossmodal attentional blink between vision and touch. Psychonomic Bulletin \& Review, 9, 731738.

VAN SELST, M., \& Joliceur, P. (1994). A solution to the effect of sample size on outlier elimination. Quarterly Journal of Experimental Psychology, 47A, 631-650.

Vogel, E. K., LucK, S. J., \& ShapIro, K. L. (1998). Electrophysiological evidence for a postperceptual locus of suppression during the attentional blink. Journal of Experimental Psychology: Human Perception \& Performance, 24, 1656-1674.

WARD, R., DUNCAN, J., \& SHAPIRO, K. [L.] (1997). Effects of similarity, difficulty, and nontarget presentation on the time course of visual attention. Perception \& Psychophysics, 59, 593-600. 


\section{NOTES}

1. It is possible that target accuracy depends not only on how easily the participant can process the target, but also on the resources that the participant invests in processing that target (see McLaughlin et al., 2001). To the extent that higher target accuracy is the result of greater resource investment, one may, instead, expect a positive relationship between $\mathrm{T} 1$ accuracy and $\mathrm{AB}$ magnitude and a positive relationship between singletarget accuracy and $\mathrm{AB}$ magnitude.

2. Pilot testing was used for both single- and dual-task RSVP programs to achieve presentation durations that resulted in approximately $70 \%$ detection accuracy for target present/absent judgments.

3. Although luminance and contrast were equivalent for all letters, digits, and objects in the present experiment (all were presented in black), luminance and contrast differed element to element in the color RSVP streams. The additional luminance and contrast information may have been used more or less effectively by at least some participants, thus differentiating color RSVP scores from the letter, digit, and object scores.

4. When conditionalized $\mathrm{AB}$ magnitude scores were used as the criterion in all of the regression analyses, instead of unconditionalized $\mathrm{AB}$ magnitude scores, the pattern of results remained the same, except that T1 accuracy was no longer a significant predictor. When all non-RSVP tasks were used as predictors of conditionalized AB magnitude, they explained just over $8 \%$ of the variability in AB magnitude $(p>.63)$, with no significant unique predictors. When T1, T2, and single-target accuracy were added, just over $11 \%$ of the variability in $\mathrm{AB}$ magnitude was explained, again with no significant unique predictors. The overall $\mathrm{AB}$ magnitude was $30.4 \%(68.3 \%-37.9 \%)$ when conditionalizing on T1 accuracy.

(Manuscript received September 27, 2004; revision accepted for publication July 19, 2005.) 